\title{
Developing an IoT Platform for the Elderly Health Care
}

\author{
Medhat Awadalla ${ }^{1}$, Firdous Kausar ${ }^{2}$, Razzaqul Ahshan ${ }^{3}$ \\ Department of Electrical and Computer Engineering \\ College of Engineering, Sultan Qaboos University \\ Muscat, Oman
}

\begin{abstract}
The health care of elderly people addresses the necessity for services that utilize recent technologies and devices. Now-a-days, both loneliness and psychological depressions are typical problems which elderly people face because of living alone/abandoned or reduced communication with their children and relatives. This paper presents the development of an integrated platform using the Internet of Things to manage and provide extensive services for elderly people to address the aforementioned issues. The proposed platform relies on wearable sensor devices to collect real-time data and store it in a cloud server via a developed smartphone application. The cloud server is accessed to retrieve data stored using the OAuth protocol. A web-based database-driven application is developed that facilitates the management of helpful information about elderly people to an authorized person. The doctors can perform realtime monitoring of the health condition of their elder patients remotely, and the system generates an alarm and sends notifications to caregivers and doctors in case of emergency. The conducted experiments and the achieved results showed that the developed platform has remarkable features of accessibility, security, efficiency, and cost.
\end{abstract}

Keywords-Internet of things; healthcare; sensor network; real-time monitoring; wearable sensors; wireless sensor networks

\section{INTRODUCTION}

Nowadays, the number of elderly is growing up, and it is expected to reach from 962 million globally in 2017 to 2.1 billion in 2050 [1]. Many elderly people lose their strength and ability to serve themselves if they are living alone. However, many of the elderly live alone in some societies since their children leave their homes when they reach the age of 18th. In Oman, the whole family lived together in the same village in the past because most of them worked in the same area, so there was no problem taking care of such issues. Nowadays, due to the lack of jobs in villages, people leave their villages and go to cities for jobs, which most probably be very away from their homes. Consequently, this will cause problems in monitoring their elderly health.

It is a global concern, and many of the elderly have numerous health problems such as hypertension and hypothesis, diabetes, heartbeat problems, and much more. Such illnesses need to be monitored to ensure no risk of death due to any sudden changes. In some countries, like Oman, it is impossible to leave the elderly to stay in some organizations like "Elderly Home" to monitor their health; since it is considered ingratitude, the same situation for all other Arabian countries. The hospitals cannot monitor all the elderly people constantly inside their buildings due to the limitations of medical staff and beds. Furthermore, the escorts cannot regularly stay in the hospital since they have other work to do, especially if it is not an emergency case that requires the elderly people to stay at the hospital. Moreover, when people become old, their brains do not have the same ability as adults in remembering things, which means that they may forget how to get back to the house if they go outside. The relatives will face problems finding them, and the risk will increase if the elderly suffer from any diseases.

Therefore, a system is proposed which enables continuous monitoring for elderly people's health in real-time to prevent chronic diseases, thus preventing hospitalization that burden the healthcare systems and costs. The proposed system measures some parameters and monitors the elderly people at home or in a particular area outside the home. This system works automatically using wireless communication and also monitors heartbeat, steps, and calories. It gives an alert to the hospital and caregivers when a problem occurs. Also, the health data information of elderly people will be regularly sent and stored in a database.

Furthermore, the system will display all elderly information on a website. Therefore, it will be easy for doctors and relatives to monitor their health status. Since the relatives may be far away from the elderly at the emergency case, the doctors can access the door directly from the website even if it is locked to save time.

The rest of the paper is organized as follows: related work for the proposed system is discussed in Section 2. The proposed system architecture is described in Section 3. The developed database system is presented in Section 4. The system implementation and results are presented in Section 5. Finally, the paper is concluded in Section 6 with a potential for future directions of this work.

\section{RELATED WORK}

Much work has been conducted to address remote health monitoring and develop systems committed to helping elderly people who live in isolated regions. Wearable devices have contributed to the development of non-invasive strategies to monitor people's activities. Authors in [2] proposed a smart home for elderly care based on a wireless sensor network to monitor and facilitate the elderly. This system integrates many sensors intending to get the required measured parameters: fire detection, gas leakage detection, and the determination of 
either door as closed or opened. Another research is proposed in an indoor system based on wireless sensor network technology capable of monitoring heart rate and motion rate. The system is competent to alert whoever cares about the elderly via a smartphone [3]. Other research is proposed to monitor the health of the elderly by measuring the arterial, electrocardiogram, saturation of respiratory oxygen rhythm, blood pressure, and body temperature, then transmits these measurements to a central medical server using Wi-Fi or GSM/GPRS connection [4]. Authors in [5] proposed a system for managing the time of taking medication and monitoring the status based on RFID and wireless sensors.

Moreover, a system that continuously monitors the health state of elderly people and enables them to contact doctors during any emergency cases has been presented $[6,18]$. The system can measure human physiological parameters such as breathing frequency, under clothing temperature, heart pulses, positioning inside and outside the house. However, the mentioned systems can monitor the status of elderly people. Some of them still rely on sensors that are not convenient for elderly people.

The industry and research group have conducted projects in health care, for instance, the project called "My-Heart" [11] that focuses on monitoring the measured heart signals to predict and assist patients who are suffering from heart diseases. Further, the project called "HeartCycle" [12, 13] issues a solution for people with Coronary Heart Disease and Heart Failure. Continua Health Alliance has also performed significant enhancement in defining interoperability for both LAN and WAN interfaces and now enables end-to-end interoperability $[14,15,17]$.

The proposed system in this paper depends on wearable devices and smartphone devices. The wearable devices are used for tracking data of physical activities. Such tools are used to improve human health by encouraging them to exercise and follow correct behavior in nutrition, sleep, and life. Based on some statistics, about $46 \%$ of people older than 65 in the United States own a smartphone [7]. Therefore, this refers to a considerable number of elderly people moving to these new technologies. Many people, especially the elderly, seek a wearable device, which monitors their health and provides essential health parameters to them and their registered doctors [8-10]. Most people are using a wearable watch for fitness, but we use it in this system for taking care of the elderly by integrating a smartwatch with a mobile phone to extract the desired data to analyze them and take actions based on them.

\section{PRoposed System ARCHITECTURE}

As shown in Fig. 1, the proposed system consists of three units: Elderly, server, and caregivers. Fitbit watch is used to measure the health parameters such as heartbeat, steps, calories, sleep, etc. The smartphone is used as a gateway to send data to the Fitbit servers, and then the data can be retrieved from the Fitbit servers to the local server. Fig. 2 shows the proposed system architecture. Here, we present the main components of the developed platform that targets elderly people monitoring. The proposed platform monitors physiological data of the elderly (e.g., heart rate), as shown in Fig. 1. A wearable device is used, Fitbit is used to collect real- time data and store it in the Fitbit cloud server via a smartphone. This data will be retrieved using the "OAuth" protocol through API requests.

The developed system comprises a web-based databasedriven application that facilities the sharing and management of useful information about elderly people to an authorized person. The developed system can open the front door of accommodation of older people in case of emergency remotely, provided that caregivers are unreachable. Simply, there is an interfacing action between the doctors and elderly system, see Fig. 1. The block diagram of the proposed system architecture is presented in Fig. 2.

Algorithm 1: System workflow

Output: Elderly status monitoring.

do

\{

if (a new elderly is registered)

then

\{

Assign doctors for the elderly

Register relatives, their locations.

Collect information and update the hospital database server \}

else

\{

if (A service is required for the elderly) then

\{

Doctors access the website server to monitor the elderly status

if (The status is normal)

Doctor contacts and sends elderly information

else if (The status is critical)

The hospital determines the location of the nearest relative.

Doctors contacts the nearest relative

Send relatives the elderly status

\}

If (no relative reply

The hospital accesses the elderly house directly

\}

\}

while (true)

The used Communication Protocol is known as "OAuth," which is an open-standard authorization protocol or framework that provides applications for "secure designated access [9]. Password data is not sharable if the "OAuth" protocol is used. However, authorization tokens are used to differentiate among both consumers and service providers. "OAuth" protocol is an authentication protocol that allows one to approve one application interacting with another on your behalf without giving away any password. As shown in Fig. 3, it is used for user authorization and API authentication to retrieve the information of the elderly in the Fitbit server to the local server.

The developed website inside the caregiver unit is an interface among sensors readings, doctors, elderly, relatives, and the site map shown in Fig. 4. As shown in Fig. 5, the home page is divided into three sections: login, get started, and 
supported platforms. The administrators, elderly, and doctors can use the login section as depicts in Fig. 6 to log in to their pages. The elderly who wants to get started with the developed system can apply a request through the get started section as illustrated in Fig. 6. Then, the elderly will receive an email to confirm that his request has been received, and the administrator of the system will contact him and ask for the required information. The last section is the supported platform, where the people can find the platforms from anywhere, and they can buy products to integrate them with our system.

After the user logs in, he will be redirected automatically to his page. On the admin page, as shown in Fig. 7, there are web site's statistics, which show the number of doctors, the elderly, and the total number of relatives assigned to the existing elderly. Moreover, the admin can view, add, edit, and delete any existing entities on the website. Besides, he can see the elderly list of those who have not been assigned to any doctor and assign them to the proper doctor. Besides, he can reassign any of the elderly to another doctor from one previously assigned to him. The last section is the messages section. The admin can see the requests from the general people who want to use our system for the elderly. Also, he can see the problem started from the website's users.

The elderly page can be accessed by the elderly himself or by the doctor who has been assigned to him. They can see a live preview of the sensor's readings and graphs, which demonstrate the behavior of each measured parameter. Furthermore, they can generate a full report of elderly health history and print it later to use it wherever needed. Moreover, the doctor can ask the elderly to come to the hospital for a checkup if needed. In case of emergency, he can open/close the door of the elderly house to get the elderly to the hospital if the relatives do not respond to that case, see Fig. 1. On the other hand, the elderly/relative can post questions related to the health status of the elderly to get the answers from the doctor directly via the developed website. Furthermore, there is a record on the elderly page for security issues that shows the name, date, and time of anyone open/close the door from the website.

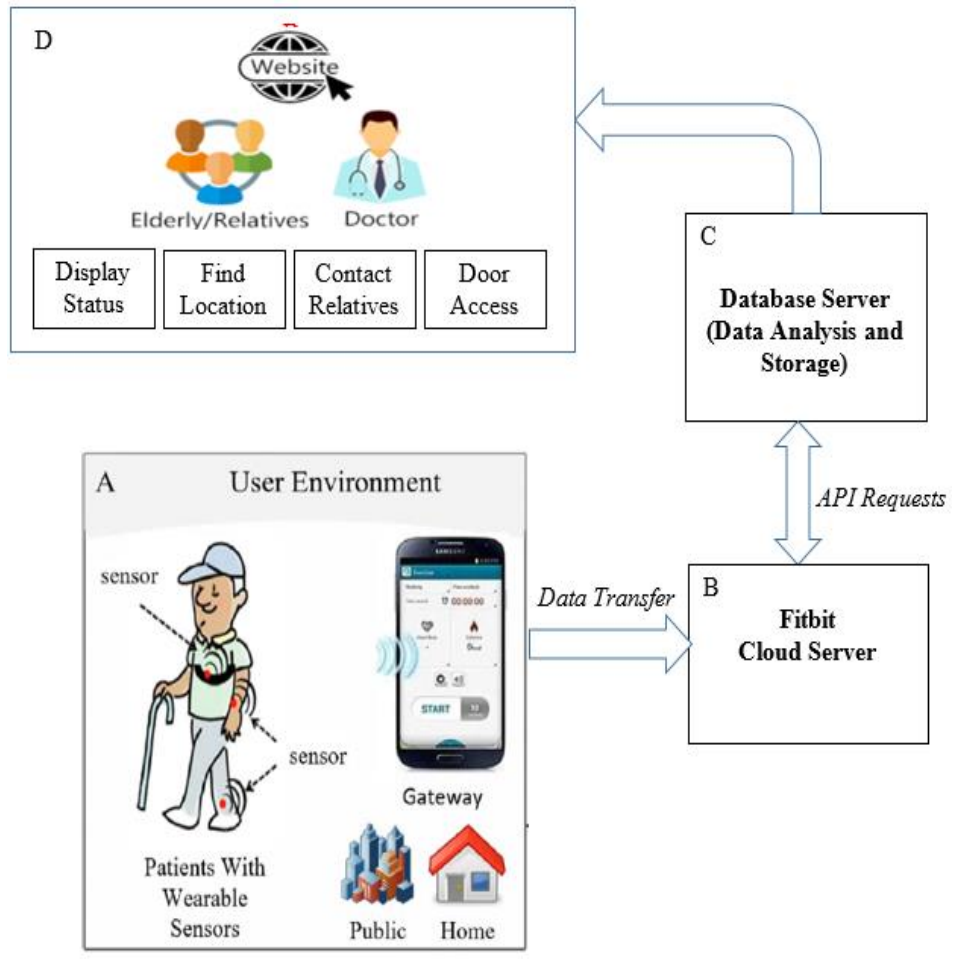

Fig. 1. The Proposed System Overview.

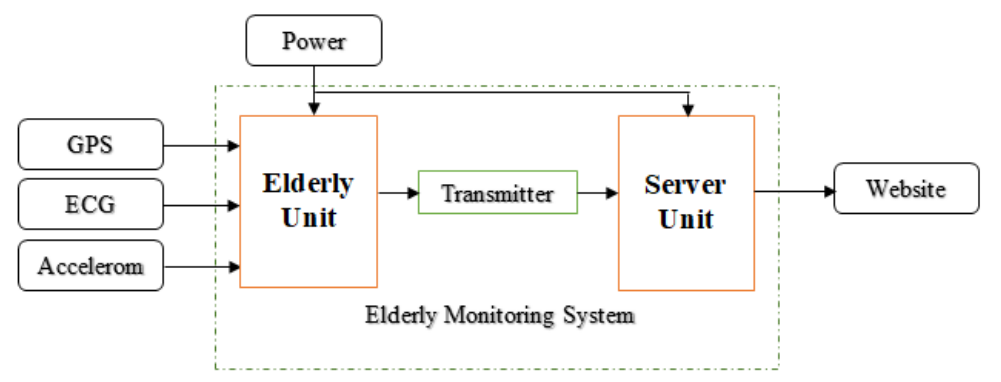

Fig. 2. The Block Diagram of Proposed System Architecture. 


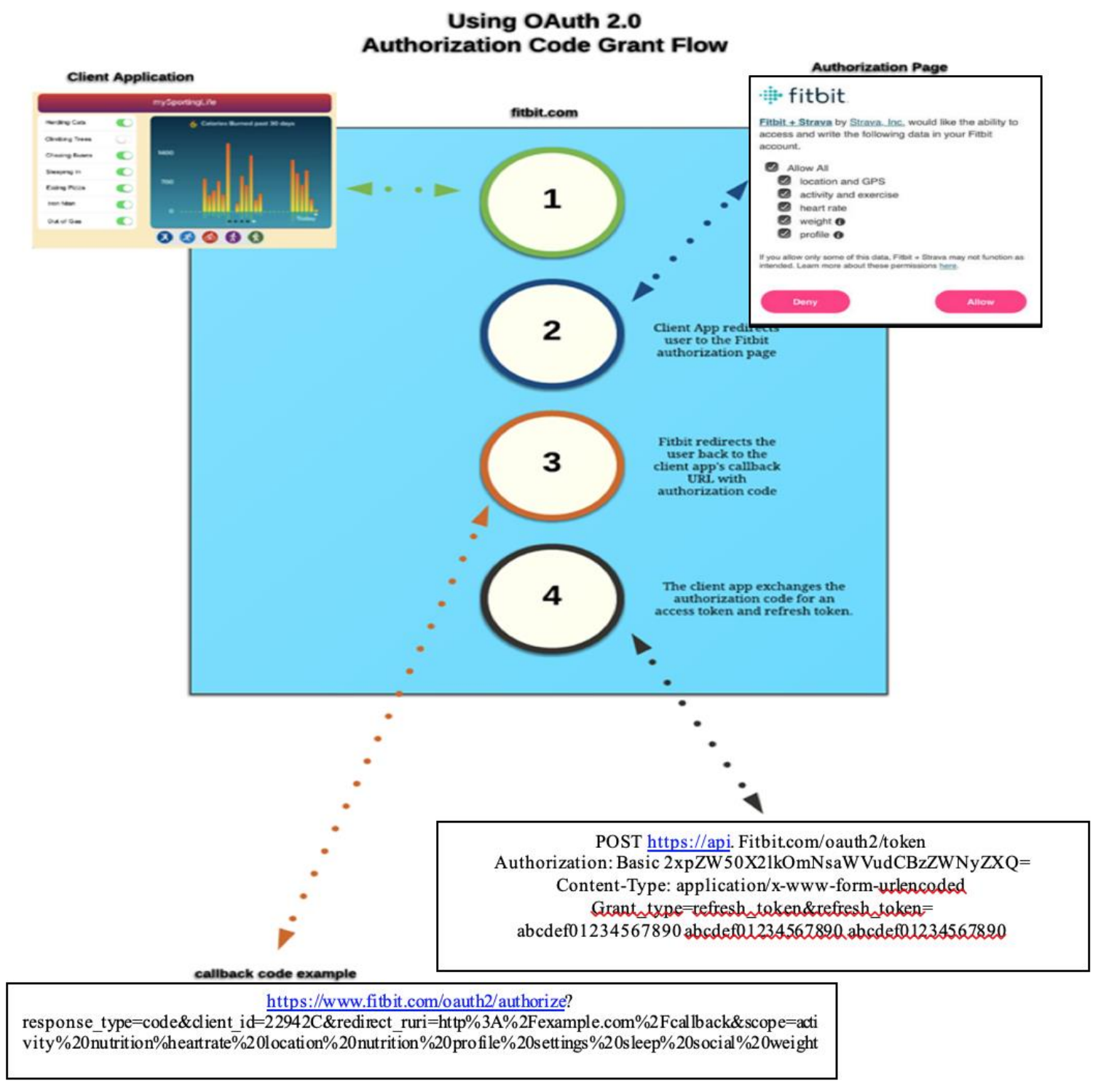

Fig. 3. OAuth 2.0 Protocol in Fitbit [9].

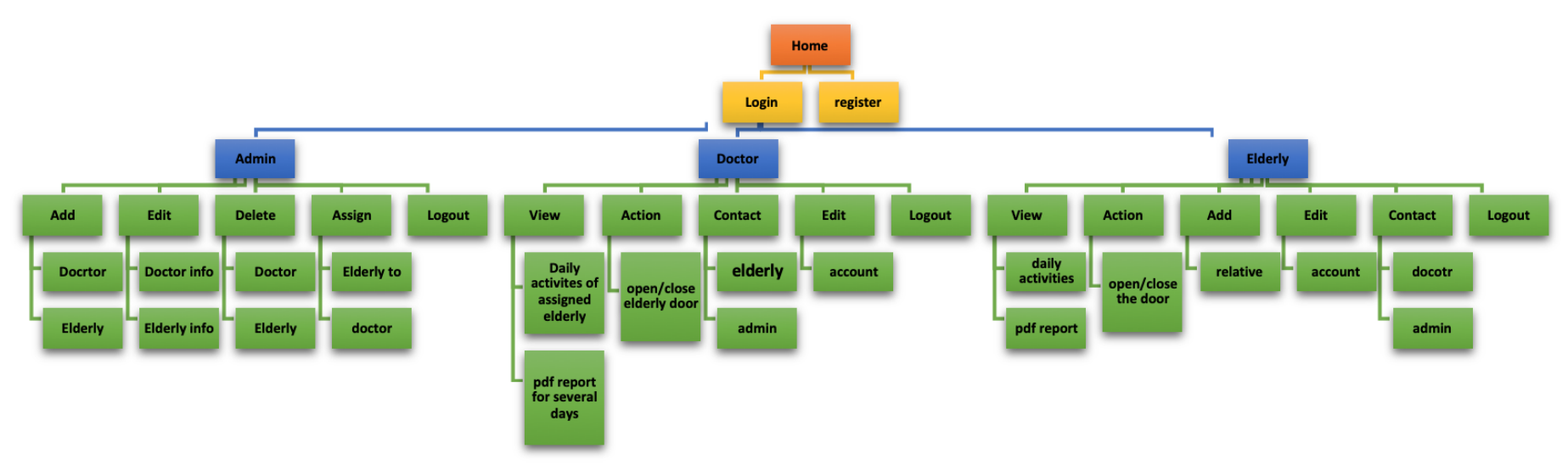

Fig. 4. The Developed Website Map. 


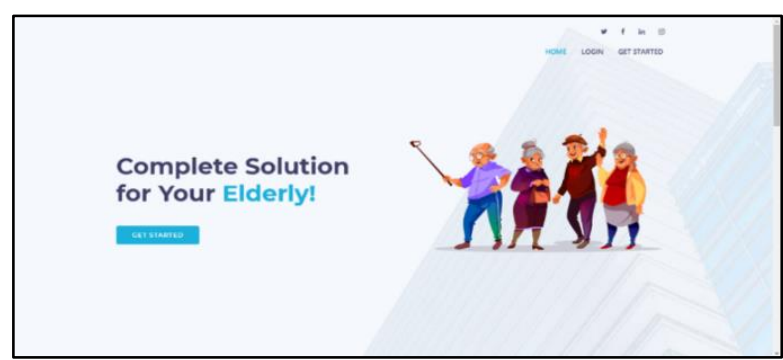

Fig. 5. The Developed Home Page.

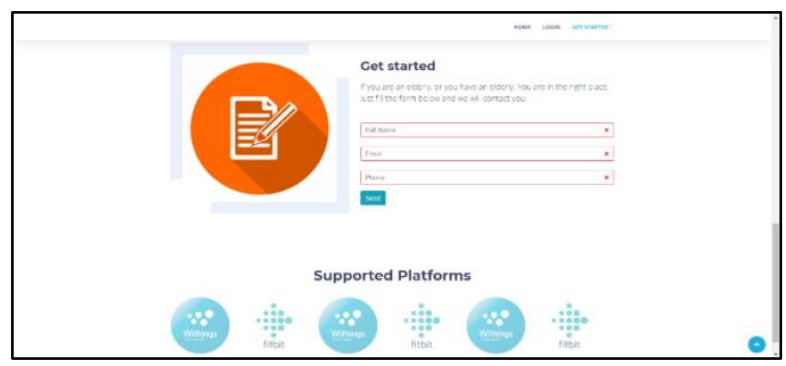

Fig. 6. Login and Support Sections on the Home Page.

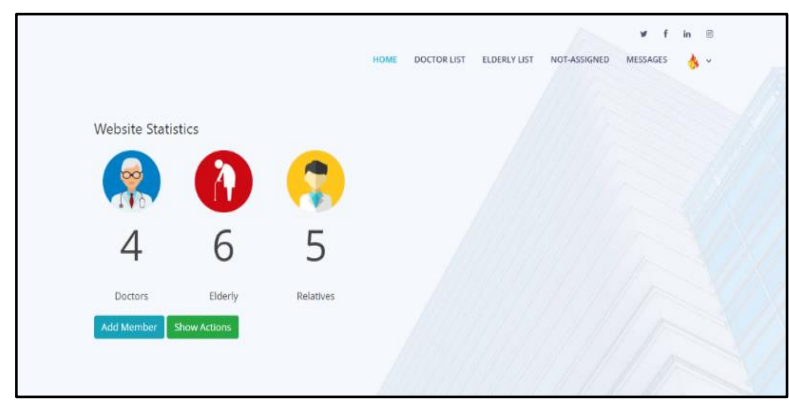

Fig. 7. The Developed Admin Page.

\section{Database Manipulation}

The database is a collection of organized information so that it can be easily accessed, managed, and updated. Data is represented in the database as tables, and it is indexed to find the relevant information easily. In this study, the database is used to store elderly readings and share them with doctors and relatives through a website [16]. The Entity-Relationship (ER) diagram of the database is shown in Fig. 8.

The business rules describe a policy, procedure, or principle within a specific organization [17-18]. It determines the entities and their relationships on the database. The business rules of the database of the proposed system are presented in Table I.

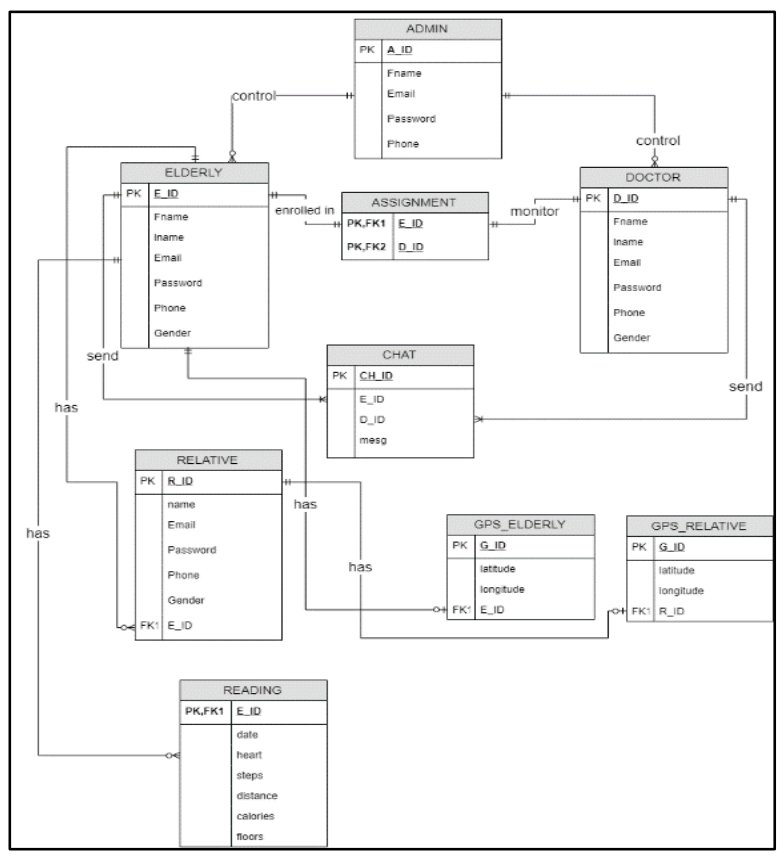

Fig. 8. The Developed ER Diagram.

TABLE I. The DATABASE Business RULES OF THE PRoPosEd System

\begin{tabular}{|l|l|}
\hline Steps & Action \\
\hline $\mathbf{1}$ & $\begin{array}{l}\text { The elderly have one relative only, but the relative may have many } \\
\text { elderly. }\end{array}$ \\
\hline $\mathbf{2}$ & $\begin{array}{l}\text { The doctor may monitor one or more elderly, but each elderly is } \\
\text { monitored by one doctor. }\end{array}$ \\
\hline $\mathbf{3}$ & The elderly store data in history. \\
\hline $\mathbf{4}$ & The Elderly can chat with his doctor. \\
\hline $\mathbf{5}$ & GPS locates the elderly and relatives. \\
\hline $\mathbf{6}$ & Each elderly has a health parameter reading. \\
\hline
\end{tabular}

\section{IMPLEMENTATION}

To read the Fitbit watch parameters (heartbeat, steps, calories, sleep, etc.), the Fitbit watch should be connected to the phone using Bluetooth communication. The mobile phone is used as a gateway between the Fitbit watch and the server unit. Fig. 9 shows the data that is read by the Fitbit watch and synchronized with the phone correctly. After uploading the data to the Fitbit server, it should be retrieved and stored in a system database. To retrieve the data, the "OAuth 2.0" protocol (Open Authentication) is used to grant Fitbit and retrieve the required data. This process requires a PHP script that is working continuously. Fig. 10 shows how the script is running and retrieving the data. 


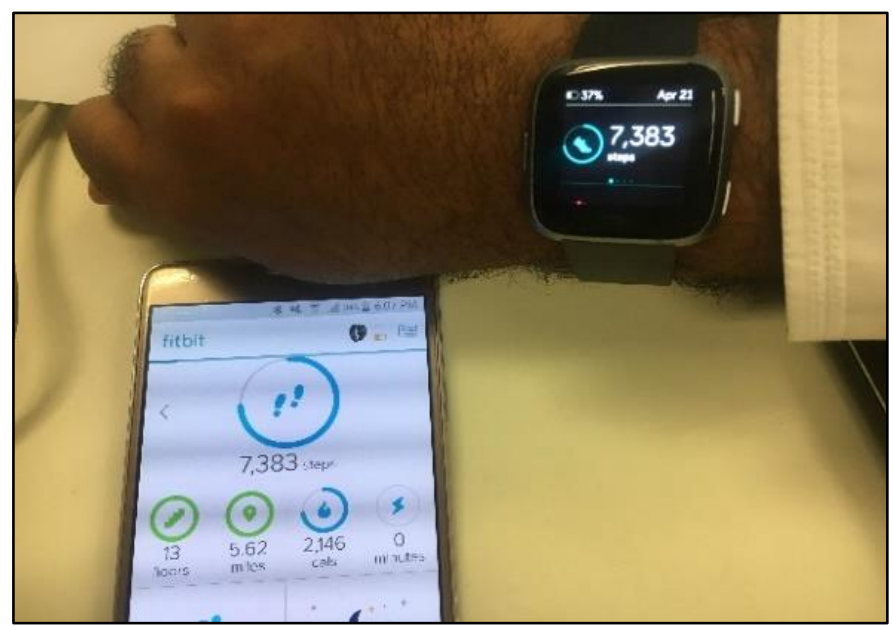

Fig. 9. The Connection of Fitbit Watch with the Mobile Phone.

\begin{tabular}{|l|}
\hline \multirow{2}{*}{ localhost/fitbit/get_daily_data.ph $\times \quad+$} \\
$\#$ C $\quad$ (i) localhost/fitbit/get_daily_data.php \\
337.88 \\
7 \\
6419 \\
$2019-04-25$ \\
$2019-04-25$ T17:08:15.992
\end{tabular}

Fig. 10. Get Daily Data Script.

Fitbit provides 150 requests for each user per hour, which forces the designer to manage the requests based on the need for data. For example, daily data will be requested every 3 minutes, so this will cost 50 requests, and the remaining 100 requests will be used to check the connection used with daily activity data, so the remaining is about 50 requests. The left requests will be used to get heart rate data. Elderly and their relatives can contact the doctors through the website; for example, if they need some help or to ask the doctor about an appointment. When an elderly or relative sends a message to the doctor, the message will be saved in the database, and then the message will be sent to the doctor and vice versa (see Fig. 11.

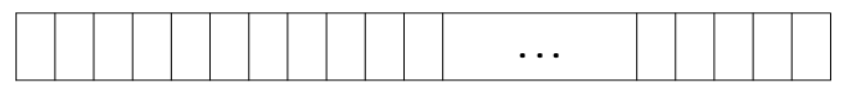

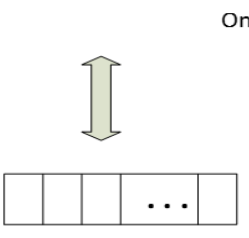

Fifty requests for heart rate data/h
One hundred and fifty requests for each user/h

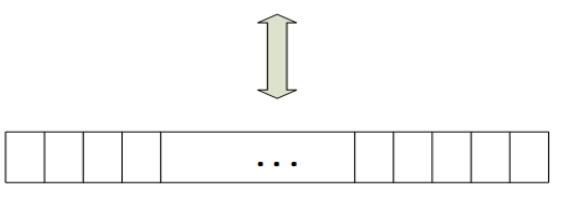

A hundred requests for connection check/h
Fig. 11. Requests Provided for users using Fitbit.
Elderly or relatives can contact doctors through the website. For example, if the elderly need some help or needs to ask the doctor about an appointment. The message will be written, saved in the database, and then sent to the doctor and vice versa. The website can add doctors, elderly, and elderly's relatives by the admin that can manage the website. The admin only can add, edit, and remove the user's account, as shown in Fig. 12.

In case of emergency, the medic wants to enter the elderly home, and he needs to control the door of the elderly house. If the door is closed, he needs to open it. By adding a smart lock to the door of the elderly home, doctors can open and close the door remotely from the website. The developed systems support tracking the elderly location if he goes outside the house. The system also calculates, and the doctor can determine the nearest relative to the elderly and sends a notification to him/her.

Fig. 14 shows the location of the elderly, while the blue point and the red points refer to the relatives. Furthermore, a PHP script using Twilio API is implemented to call the relative of the elderly whenever an emergency case occurs. This should work automatically using phone call as shown in Fig. 13.

The integrated system was tested, and the results are shown in Table II. Fig. 12 to 15 shows the system outputs, the recorded heartbeat; Fig. 16 shows the measured data of different activities of the elderly. Fig. 17 gives the statistical data collected during a month.
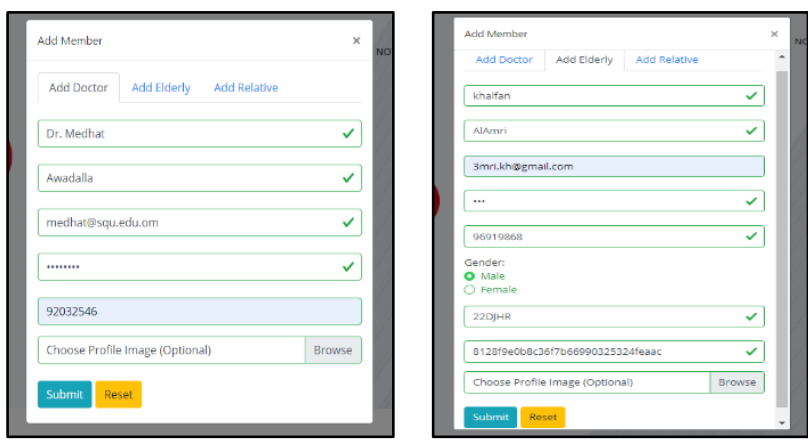

Fig. 12. Adding Doctor and Elderly.

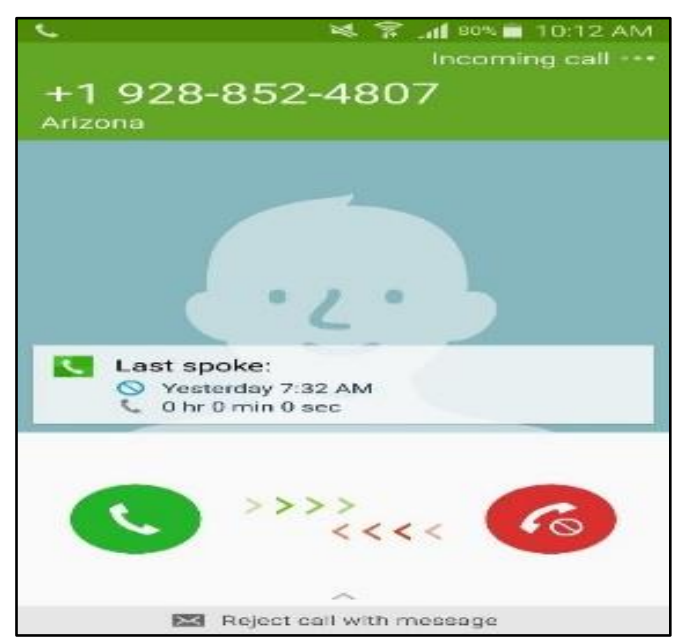

Fig. 13. Track Elderly Location based on GPS Navigator. 


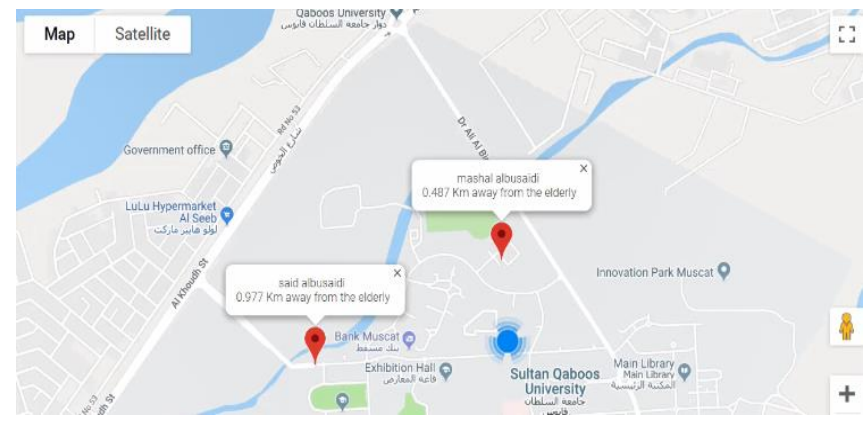

Fig. 14. Contacting the Relative of the Elderly.

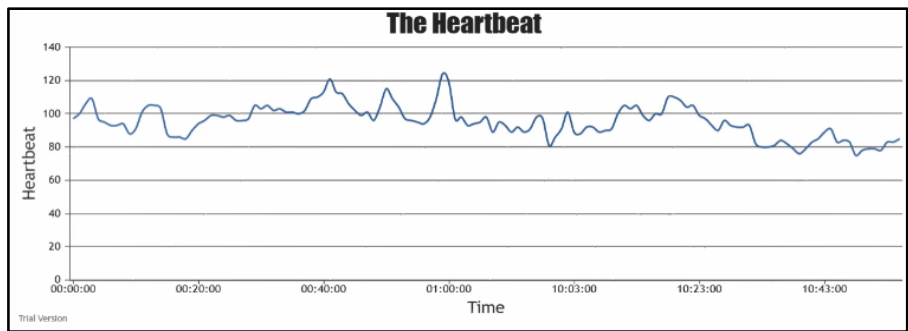

Fig. 15. The Recorded Elderly Heartbeat.

TABLE II. INTEGRATION TEST FOR THE WHOLE SYSTEM

\begin{tabular}{|c|c|c|c|c|c|}
\hline \multicolumn{6}{|c|}{$\begin{array}{l}\text { Test name: Integration for the whole system } \\
\text { Test ID\#: IT-01 }\end{array}$} \\
\hline \multicolumn{6}{|c|}{ Test description: Verify that the project is working } \\
\hline \multicolumn{6}{|c|}{ Test information } \\
\hline \multicolumn{2}{|c|}{ Name of tester: ALL } & \multicolumn{3}{|c|}{ Date: $25 / 2 / 2021$} & $\begin{array}{l}\text { Time: } \\
\text { 2:00 PM }\end{array}$ \\
\hline$\#$ & Procedure & Pass & Fail & N/A & Comments \\
\hline 1 & $\begin{array}{l}\text { Watch reads all } \\
\text { parameters. }\end{array}$ & $\sqrt{ }$ & & & \\
\hline 2 & Watch sends the data. & $\sqrt{ }$ & & & \\
\hline 3 & $\begin{array}{l}\text { The database receives the } \\
\text { data from the watch. }\end{array}$ & $\sqrt{ }$ & & & \\
\hline 4 & $\begin{array}{l}\text { The website displays the } \\
\text { retrieved data }\end{array}$ & $\sqrt{ }$ & & & \\
\hline 5 & $\begin{array}{l}\text { Tracking the live location } \\
\text { of the elderly. }\end{array}$ & $\sqrt{ }$ & & & \\
\hline 6 & $\begin{array}{l}\text { Measure the distance } \\
\text { between the elderly and } \\
\text { relatives. }\end{array}$ & $\sqrt{ }$ & & & \\
\hline 7 & $\begin{array}{l}\text { Send SMS alarm } \\
\text { automatically if there is } \\
\text { an emergency. }\end{array}$ & $\sqrt{ }$ & & & \\
\hline 8 & $\begin{array}{l}\text { Call relatives } \\
\text { automatically if there is } \\
\text { an emergency. }\end{array}$ & $\sqrt{ }$ & & & \\
\hline 9 & $\begin{array}{l}\text { Open and close the door } \\
\text { from the website. }\end{array}$ & $\sqrt{ }$ & & & \\
\hline
\end{tabular}

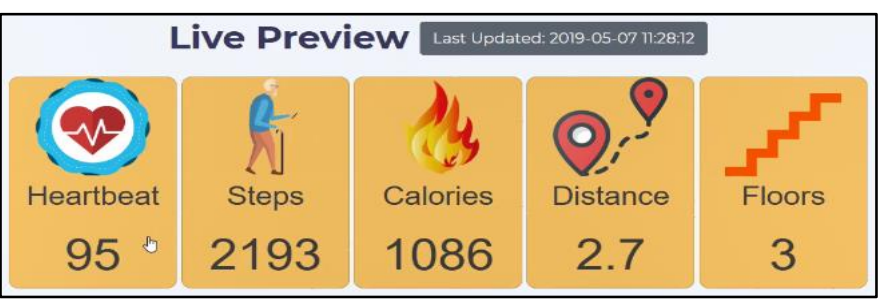

Fig. 16. The Measured Data of the Elderly Activities.

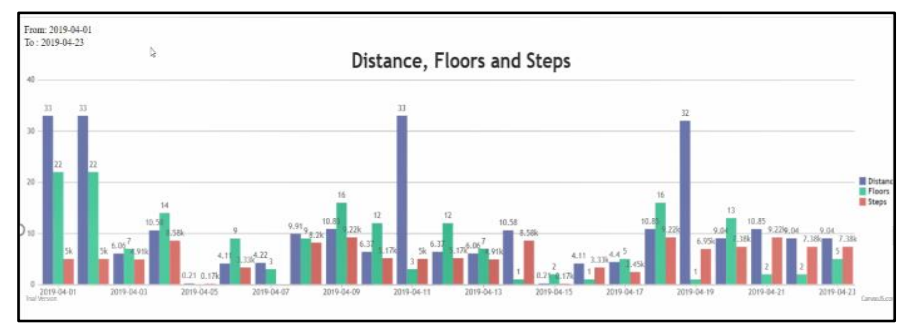

Fig. 17. The Statistical Data of the Elderly.

\section{CONCLUSION}

In this paper, an integrated IoT-based platform is presented to monitor and collect vital data from elderly people. The developed platform relies on wearable devices and smartphones. Fitbit is used to collect real-time data and store it in the Fitbit cloud server via a smartphone. OAuth protocol is used to retrieve data stored in the Fitbit cloud server. A webbased database-driven application is developed that facilities the management of helpful information about elderly people to an authorized person. The stored data is used for analysis, which helps the doctors track their patients' evolution. A complete platform to verify the idea is implemented and tested to validate the system functionality. The results show promising outcomes for monitoring elderly people. Besides, the features of the developed system increase accessibility, efficiency and lower the health expenses that improve the comfortability and safety as well as management of daily routines.

\section{ACKNOWLEDGMENT}

The Sultan Qaboos University supports the work in this paper under the internal grant approved for the research project code number IG/ENG/ECED/21/01. Moreover, we are grateful to the anonymous reviewers for their valued feedback.

\section{REFERENCES}

[1] Aging, United Nations, Levels and trends in Population Aging, Available from URL:http://www.un.org/en/sections/issues-depth/ageing last update [6/02/2021].

[2] Rasika S. Ransing, Manita Rajput; Smart Home for Elderly Care, based on Wireless Sensor Network, International Conference on Nascent Technologies in the Engineering Field, 2015, p.p.1-5, 2015.

[3] Pedro Magaña-Espinoza, Raúl Aquino-Santos, Néstor Cárdenas-Benítez, José Aguilar-Velasco; A Wireless Sensor Network-Based Home Care Monitoring System, IEEE Sensors Journal 12(6):1965-1972, 2012. 
[4] C. Rotariu, H. Costin, Gladiola Andruseac, R. Ciobotariu, F. Adochiei: C. Rotariu, H. Costin, Gladiola Andruseac, R. Ciobotariu, F. Adochiei, "An Integrated System for Wireless Monitoring of Chronic Patients and Elderly People". Proc. of the 15th IEEE Int. Conference on System Theory, Control and Computing, ICSTCC2011, Sinaia, Romania, pp. 527-530, 2011.

[5] ZigBee Vs. XBee: An Easy-To-Understand Comparison, Available from URL: https://www.link-labs.com/blog/zigbee-vs-xbee , Last updated [15/1/2021].

[6] Ching-Nung Yang, Fu-Heng Wu, Sin-Yen Tsai, Wen-Chun Kuo: EHealth Services for Elderly Care Based on Google Cloud Messaging. IEEE International Conference on Smart City/SocialCom/SustainCom together with DataCom 2015 and SC2 2015, pp. 9-12, 2015.

[7] Michal Frydrysiak, Lukasz Tesiorowski. Wearable textronic system for protecting elderly people. IEEE International Multidisciplinary Conference on Computer and Energy Science (SpliTech), p.p.1-6, July 2016.

[8] Mircea Serbanescu, V. M. Placinta, O. E. Hutanu, Cristian Ravariu. Smart, low power, wearable multi-sensor data acquisition system for environmental monitoring. 2017 10th International Symposium on Advanced Topics in Electrical Engineering (ATEE), 2017.

[9] Mostafa Haghi, Regina Stoll, Kerstin Thurow. A Low-Cost, Standalone, and Multi-Tasking Watch for Personalized Environmental Monitoring. IEEE Transactions on Biomedical Circuits and Systems, 2018.

[10] Luprano J, Sol'a J, Dasen S, Koller JM, Ch'etelat O (2016) Combination of body sensor networks and on-body signal processing algorithms: the practical case of MyHeart project. In: null. IEEE, pp 76-79.
[11] Chouvarda I, Antony R, Torabi A, Weston J, Caffarel J, van Gils M, Cleland J, Maglaveras N (2013) Temporal Variation in telemonitoring data: on the Effect of Medication and Lifestyle Compliance. International Journal of Bioelectromagnetism.

[12] Palumbo F, Ullberg J, timec A, Furfari F, Karlsson L, Coradeschi S (2014) Sensor network infrastructure for a home care monitoring system. Sensors.

[13] Wartena F, Muskens J, Schmitt L, PetkovicM(2010) Continua: The Reference Architecture of a Personal Telehealth Ecosystem. In: proceedings of 12th IEEE international conference on e-health networking applications and services (Healthcom), Lyon, France.

[14] Meredith A. Case, Holland A. Burwick, Kevin G. Volpp, Mitesh S. Patel. Accuracy of Smartphone Applications and Wearable Devices for Tracking Physical Activity Data. JAMA February 10, 2015 Volume 313, Number 6, pp. 625-626, 2015.

[15] Mobile Fact Sheet, Who owns cellphones and smartphones, Available from URL: https://www.pewinternet.org/fact-sheet/mobile/ Last updated [10/2/2021].

[16] C. Coronel, S. Morris, and P. Rob. Database Systems: Design, Implementation, and Management, Boston, Ninth Edition, 2011.

[17] Zhou, Jining; Zhang, Bo; Tan, Runhua; Tseng, Ming-Lang; Lin, Remen C.-W.; Lim, Ming K. 2020. "Using Neighborhood Rough Set Theory to Address the Smart Elderly Care in Multi-Level Attributes" Symmetry 12, no. 2: 297.

[18] Selvaraj, S., Sundaravaradhan, S. Challenges and opportunities in IoT healthcare systems: a systematic review. SN Appl. Sci. 2, 139 (2020) 